\title{
Entanglement of midspectrum eigenstates of chaotic many-body systems: Reasons for deviation from random ensembles
}

\author{
Masudul Haque $\odot,{ }^{1,2,3}$ Paul A. McClarty, ${ }^{2}$ and Ivan M. Khaymovich $\odot^{2,4}$ \\ ${ }^{1}$ Department of Theoretical Physics, Maynooth University, County Kildare, Ireland \\ ${ }^{2}$ Max-Planck-Institut für Physik komplexer Systeme, D-01187 Dresden, Germany \\ ${ }^{3}$ Institut für Theoretische Physik, Technische Universität Dresden, D-01062 Dresden, Germany \\ ${ }^{4}$ Institute for Physics of Microstructures, Russian Academy of Sciences, 603950 Nizhny Novgorod, GSP-105, Russia
}

(Received 17 September 2020; accepted 14 December 2021; published 7 January 2022)

\begin{abstract}
Eigenstates of local many-body interacting systems that are far from spectral edges are thought to be ergodic and close to being random states. This is consistent with the eigenstate thermalization hypothesis and volume-law scaling of entanglement. We point out that systematic departures from complete randomness are generically present in midspectrum eigenstates, and focus on the departure of the entanglement entropy from the randomstate prediction. We show that the departure is (partly) due to spatial correlations and due to orthogonality to the eigenstates at the spectral edge, which imposes structure on the midspectrum eigenstates.
\end{abstract}

DOI: 10.1103/PhysRevE.105.014109

\section{INTRODUCTION}

Ergodicity and equilibration in the quantum realm remain imperfectly understood, and the characterization of quantum ergodicity is now an active research front. One view is that quantum ergodicity corresponds to eigenstates of manybody systems being effectively random. This idea is closely connected to the eigenstate thermalization hypothesis (ETH) [1-7] and to ideas loosely known as (canonical) typicality [7-18]. For a nonintegrable (chaotic) many-body Hamiltonian $H$, it is expected that a state $\left|\psi_{R}\right\rangle$ with independent Gaussian random coefficients should be a good model for infinite-temperature eigenstates, while eigenstates at energy corresponding to temperature $1 / \beta$ should be well-described by $e^{-\frac{1}{2} \beta H}\left|\psi_{R}\right\rangle$ [17,19-22]. This expectation is mirrored by the behavior of the entanglement entropy (EE) in eigenstates of many-body systems with finite Hilbert spaces: At the spectral edges, EE is low ("area law") [23,24], while in the infinitetemperature (midspectrum) regime, the eigenstates have EE close to the value expected for random states. As a result, for chaotic many-body systems, the scatter plot of EE versus eigenenergy takes the shape of an arch or rainbow, by now familiar from many numerical examples [25-37].

In this work, we consider the bipartite entanglement entropy of midspectrum eigenstates. For definiteness, we focus on spin-1/2 chains with $L$ sites with all symmetries broken, so that the Hilbert space is $D=2^{L}$, and consider the entanglement between two subsystems $(A, B)$ of equal size.

Published by the American Physical Society under the terms of the Creative Commons Attribution 4.0 International license. Further distribution of this work must maintain attribution to the author(s) and the published article's title, journal citation, and DOI. Open access publication funded by the Max Planck Society.
In this case, the random states have an average EE wellapproximated by the Page formula [38,39], $S_{\text {Page }}=\log D_{A}-$ $\frac{1}{2}$, where $D_{A}=2^{L / 2}$ is the size of the reduced Hilbert space of the $A$ subsystem. Although the midspectrum eigenstates are expected to be random, numerically calculated midspectrum entanglement in finite-size many-body systems-both in the existing literature $[25,40,41]$ and in this work-systematically fall below the Page value. The deviation decreases more slowly with system size than the width of the state-tostate fluctuations of EEs, which means that the deviation is

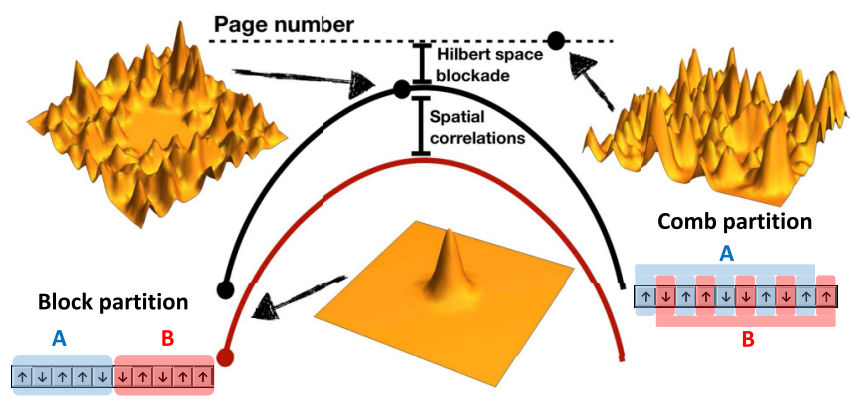

FIG. 1. Schematic, summarizing main ideas. Lower rainbow shows block EE against eigenenergies. Upper rainbow is EE between "comb" partitions. Partitions are shown in the left and right insets, respectively. The difference between the top of the two rainbows is attributed to residual spatial correlation in midspectrum eigenstates. The difference between the Page value (dotted horizontal line) and the midspectrum comb entanglement is attributed to the "orthogonality blockade" effect—orthogonality to the special spectral edge states. 3D plots are cartoons of distributions of eigenstate intensities, using a 2D space to visualize the Hilbert space. The three cases correspond to low-entanglement states typical of spectral edges (middle), fully random or "ergodic" states (right), and midspectrum states (left). The latter demonstrates a depletion of weight in parts of the Hilbert space where the spectral edges have large weight. 
significant at any finite size. To the best of our knowledge, the origin of this subtle, systematic and seemingly universal effect has not been addressed so far. In this work, we present a study of this discrepancy, uncovering the ways in which midspectrum eigenstates deviate from random states.

We find that the locality of the Hamiltonian leads to spatial correlations persisting in midspectrum eigenstates of any finite system; we demonstrate this through the mutual information between sites. The midspectrum eigenstates thus differ in an important manner from random states. The presence of spatial correlations manifests itself strongly in the entanglement between spatially connected blocks (block bipartition) - a partitioning which is naturally sensitive to spatial correlations in eigenstates. We show that the departure of midspectrum entanglement from the Page value is smaller for comb partitions that are, of all bipartitions, the least sensitive to spatial variations of correlations. Nevertheless, even the comb entanglements depart from the Page value. We argue that the reason for the departure from Page value of the midspectrum eigenstates is their orthogonality to the eigenstates at spectral edges. Orthogonality forces the midspectrum eigenstates to live in an effectively lower-dimensional Hilbert space: part of the physical Hilbert space is blocked off. This Hilbert space blockade phenomenon manifests itself in the eigenstate coefficient distribution as an enhanced weight around zero [42-45]. The orthogonality blockade effect exists for any Hamiltonian, local or not. However, for local Hamiltonians, eigenstates at the spectral edges have area law entanglement and strong spatial correlations. The blockade effect then forces midspectrum states to have the observed spatial correlations. The departure from the Page value for comb partitions is thus due to a correlated Hilbert space blockade, such that certain types of configurations in the Hilbert space are blocked from appearing in the midspectrum eigenstates. This scenario is illustrated in Fig. 1 and elaborated in the rest of this paper.

\section{MODEL}

We focus on the spin- $\frac{1}{2}$ chain, with couplings between sites $i, j$ having the $X Y Z$ form $h_{i, j}[\eta, \Delta]=(1-\eta) S_{i}^{x} S_{j}^{x}+(1+$

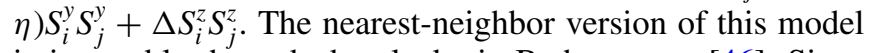
is integrable through the algebraic Bethe ansatz [46]. Since our focus is on nonintegrable systems, we add next-nearest neighbor couplings $h_{j, j+2}$ and/or magnetic fields:

$$
\begin{aligned}
H= & J_{1} \sum_{j=1}^{L-1} h_{j, j+1}\left[\eta_{1}, \Delta_{1}\right]+J_{2} \sum_{j=2}^{L-2} h_{j, j+2}\left[\eta_{2}, \Delta_{2}\right] \\
& +h_{z} \sum_{j=1}^{L}\left(1-\frac{1}{2} \delta_{j L}\right) S_{j}^{z}+h_{x} \sum_{j=1}^{L}\left(1-\frac{1}{2} \delta_{j 1}\right) S_{j}^{x} .
\end{aligned}
$$

The $h_{x}$ term breaks the parity of total- $S^{z}$. In addition, the $J_{2}, h_{z}$ and $h_{x}$ terms are each tweaked at one edge of the chain so that reflection symmetry is broken. Unless otherwise specified, we present data for $J_{\alpha}=1, \eta_{\alpha}=0.5, \Delta_{\alpha}=0.9, h_{z}=0.8, h_{x}=$ 0.2 . For parameters that we used, the level spacing statistics of the model is consistent with that of the Gaussian orthogonal ensemble (GOE), indicating chaotic behavior.
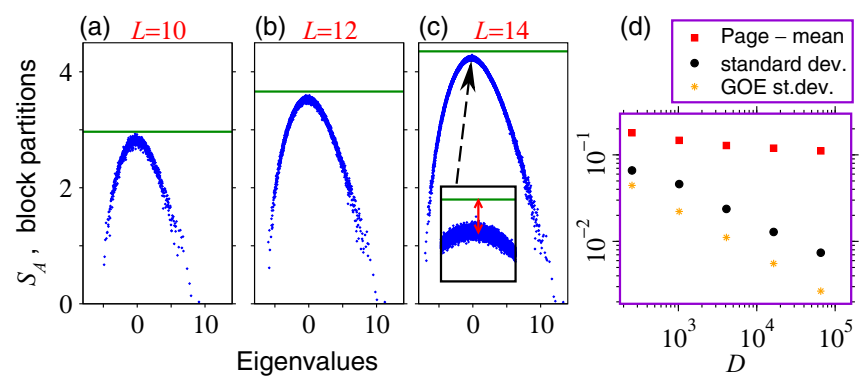

FIG. 2. EE for block bipartition. Parameters listed in text. (a-c) EE versus energy for different system sizes. Horizontal line: Page value. (d) Statistics of midspectrum eigenstates $(D / 16$ states nearest to rainbow peak), for $L=8,10,12,14,16$. The standard deviation has similar scaling $\sim D^{-1 / 2}$ to the GOE case. The departure from the Page value falls off much more slowly, possibly even saturating.

\section{ENTANGLEMENT FOR BLOCK PARTITIONS}

In Fig. 2 we consider "block" bipartitioning, i.e., the $A(B)$ bipartition is the left (right) half of the chain. The spectral edges and midspectrum states scale differently $\left(\sim L^{0}\right.$ versus $\sim L^{1}$ ), resulting in the rainbow/arch shape, Figs. 2(a)-2(c). The largest EE values are close to the EE values of random states of the same Hilbert space size, whose average is here the Page value, $S_{\text {Page }}=\frac{L}{2} \ln 2-\frac{1}{2}$, because we have chosen a spin- $\frac{1}{2}$ system with no symmetries. The EE being close to the Page value indicates that the midspectrum eigenstates are close to being "random" or "infinite-temperature." Accordingly, the width of the distribution of midspectrum EE values is expected to decrease as $\sim D^{-1 / 2}$, like eigenstates of GOE/GUE matrices [47]. Figure 2(d) shows that the midspectrum EE widths are larger than corresponding GOE values but are consistent with $\sim D^{-1 / 2}$ behavior.

The feature we focus on in this paper is the systematic departure from the Page value even in the middle of the spectrum [Fig. 2(c) inset]. Figure 2(d) shows how the departure of the mean midspectrum EE from the Page value scales with system size. There is some ambiguity in how to choose the "midspectrum" states. Figure 2(d) uses the 1/16th eigenstates closest to the top of the rainbow, but our observations are insensitive to the exact procedure [39]. The departure decreases with the system size remarkably slowly. In fact, the data does not rule out saturation, i.e., nonzero departure in the thermodynamic limit. The departure is certainly much larger than the width, which decreases much faster, $\sim D^{-1 / 2}$. Thus, at any system size, the Page value lies outside the distribution of EE values. In this sense, the departure is not "merely a finite size effect."

\section{SPATIAL CORRELATIONS AND COMB ENTANGLEMENT}

To uncover the reason for the departure from randommatrix behavior, we first appeal to the best-known case of such departures, namely, the spectral edges, for which the volume law of entanglement is violated $[23,24]$. The origin of area law EE is the spatial locality of the Hamiltonian. This causes correlations to decay rapidly with distance, and ensures that the block entanglement receives its largest contribution from the boundary region. We ask whether some degree of locality, 

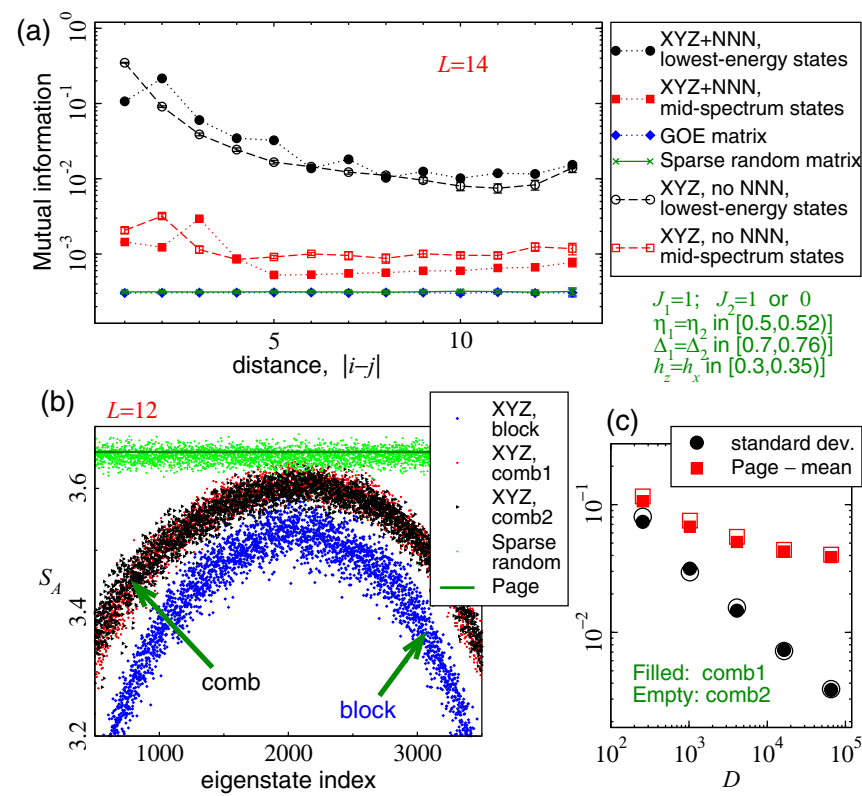

FIG. 3. Spatial correlations and comb EE. (a) Mutual information vs. distance. Low-energy and midspectrum eigenstates of $X Y Z$ Hamiltonians, compared with eigenstates of full (GOE) and sparse random matrices. Statistics gathered from 32 eigenstates of each of $40 X Y Z$ Hamiltonians, with parameters drawn from ranges shown. (b) EE for block and comb bipartitions. (c) Scaling of midspectrum comb EE (both types, filled/open symbols), similar to Fig. 2(d). The departure from the Page value falls much slower than the width.

in this sense, also exists in the midspectrum eigenstates. We quantify correlations via the quantum mutual information

$$
I(i, j) \equiv S_{[\mathrm{i}]}+S_{[\mathrm{j}]}-S_{[\mathrm{i} \cup \mathrm{j}]}
$$

for pairs of spins $i$ and $j$, as in Refs. [48,49]. Figure 3(a) shows $I(i, j)$ against the distance $|i-j|$, for midspectrum eigenstates, low-energy eigenstates and random states. The distance-dependence in midspectrum eigenstates is much less pronounced than in low-energy eigenstates, as expected. However, there exists an unmistakable dominance of smalldistance correlations. Thus, we identify distance-dependence as one reason for the departure of midspectrum EE from the Page value.

To "correct" for this effect, we consider "comb" bipartitions [50-54] of two types: "comb1" partitions spins as $A B A B A B \ldots$, i.e., it is a sublattice partition, while "comb2" partitions the spins as $A A B B A A B B$.... For comb2 partitioning, there is some ambiguity when $L$ is not divisible by 4 ; we resolve this by setting the last two sites to be in partitions $A$ and $B$. For example, for $L=10$, the comb2 partitioning is $A A B B A A B B A B$.

For such bipartitions, the EE should be insensitive to largescale distance-dependence of correlations, as two nearby points are as likely to be in different partitions as two faraway points. (The boundary and bulk of partitions are not spatially separated.) Figure 3(b) shows that the midspectrum comb EE is indeed closer to the Page value than the block EE. There is little difference between comb1 and comb2, which supports the idea that this reduction of the departure is due to removal of the effect of distance-dependence. For comb bipartitioning, there is no notion of "area law," so that the EE for spectral edges scale as $\sim L$ instead of $\sim L^{0}$. Nevertheless, the comb EE's at the spectral edges are significantly smaller than the midspectrum ones, despite having the same scaling. Thus, the comb EE's are also arranged in an arch/rainbow shape [39].

Remarkably, even for comb partitions, the departure from the Page value remains much larger than the width of midspectrum EE distributions. As in the block case, the comb EE departure decreases far slower than the width, Fig. 3(c). (The available data would even be consistent with a saturation of the departure in the $L \rightarrow \infty$ limit, as opposed to a slow decrease.) Thus, the departure is a visible effect at any finite size, also for partitions which (unlike block partitions) do not select for locality effects. We are thus forced to look for additional mechanisms-beyond "locality" as discussed above-for the departure from the random-state behavior.

\section{NONEXPLANATIONS}

\section{A. Sparsity is not responsible}

One possible source of the difference between random states and the eigenstates of local many-body Hamiltonians is that such Hamiltonians are generally sparse matrices in common basis choices. To examine the consequence of sparsity, in Figs. 3(a) and 3(b) we include results (mutual information, $\mathrm{EE})$ for the eigenstates of matrices with sparsity close to the physical (XYZ) Hamiltonian, and nonzero elements drawn from a Gaussian distribution. We find only very slight differences from eigenstates of the usual (full) GOE ensemble-in Fig. 3(a) the mutual information values for the GOE case and the sparse random case are very slightly offset from each other (offset barely visible), while in Fig. 3(b) the EE values for the sparse random matrix have a distribution whose center is only very slightly lower than the page value.

Thus, sparsity is not a significant factor in the departure from the Page value.

\section{B. Effect of finite measurement window}

To obtain sufficient statistics for the average and width of midspectrum entanglements, we use the $\mathrm{EE}$ values within some energy window, $E_{\max }-\Delta E / 2<E<E_{\max }+\Delta E / 2$, containing the energy $E_{\max }$ where the EE is maximal. Approximating the EE to be a smooth function of energy, $S(E)$, the average EE within an energy window $\Delta E$ is obtained by Taylor expansion to be smaller than the maximum by the amount $S_{\text {Taylor }}=\frac{1}{24}\left|S^{\prime \prime}\left(E_{\max }\right)\right|(\Delta E)^{2}$. We are of course interested in the limit $\Delta E \rightarrow 0$, where this effect plays no role. To ensure that we have reached this limit, we have carefully checked that our extracted values of departure are independent of the $\Delta E$ value used numerically, and also that the Taylor correction term is orders of magnitude smaller than the departure, for the values of $\Delta E$ used numerically [39].

In addition, from Fig. 2(c) inset and from Fig. 3(b), it is visually obvious that the top of the rainbow itself deviates from the Page value, and that the effect is not due to averaging over a finite energy window. 

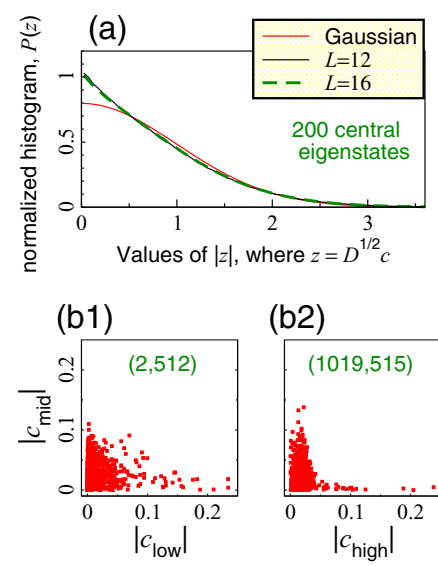

(b2)
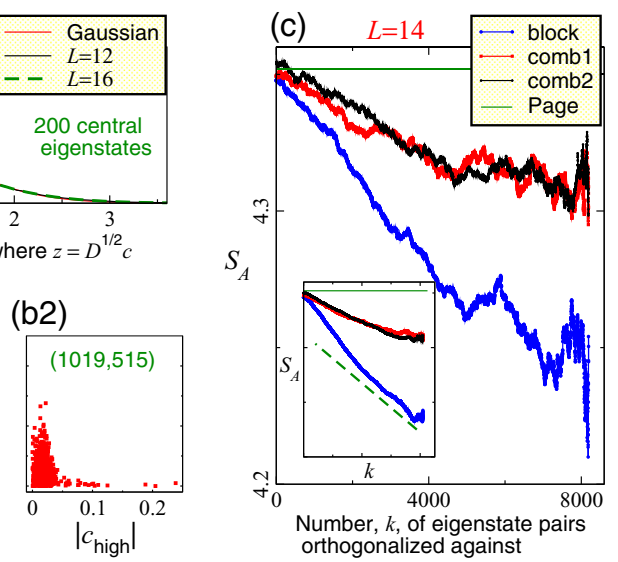

FIG. 4. Orthogonality blockade, illustrated. (a) Eigenstate coefficient distribution, showing an excess of small values compared to Gaussian. Excess appears not to scale with system size. (b) Scatterplot between eigenstate coefficients. Each point represents one real-space configuration. Coefficients of a midspectrum state against those of a low-energy (b1) and a high-energy (b2) state. Eigenstates (in brackets) labeled from 1 to $2^{10}=1024$. (c) EE of states obtained by orthogonalizing a random state to $k$ lowest-energy and $k$ highest-energy eigenstates. $k=0$ is a random state; $k=\frac{D}{2}-1$ is essentially a midspectrum eigenstate. Inset: EE's averaged over 30 starting states. Dashed straight line is a visual guide.

\section{ORTHOGONALITY AND BLOCKADE}

We now introduce a framework for discussing the deviation of midspectrum states from randomness (full ergodicity). Eigenstates at the spectral edges are well-known to be special - they have area-law instead of volume-law entanglement, and this is reflected in the local structure of correlations. These eigenstates may be seen as occupying a specific tiny part of the Hilbert space which promotes the special features. Because midspectrum eigenstates need to be orthogonal to these special states, they are forced to exclude that part of the Hilbert space. Thus, midspectrum eigenstates are distributed in a large fraction of, but not the complete, Hilbert space; part of the Hilbert space is blocked off.

This blockade phenomenon is illustrated in the cartoons of Fig. 1. If we use real-space configurations as the basis, then these cartoons over-simplify in showing the low-energy states as having exactly zero coefficients for most basis states. In reality, the low-energy eigenstates are not completely localized in configuration space-they have low but nonzero entanglement. In other words, their participation ratios [39] are much smaller than the random-state value of $D / 3$, but are still much larger than 1 [25].

Aspects of the blockade phenomenon are illustrated in Fig. 4. The coefficients of midspectrum eigenstates, in the basis of real-space configurations, are not entirely Gaussian (as would be the case for GOE eigenstates) but have excess weight at small values [42-45], Fig. 4(a). Some configurations are "over-represented" in eigenstates at the spectral edges; hence, by orthogonality they have to be under-represented in midspectrum eigenstates, leading to an excess of small values of coefficients. This effect is seen more explicitly when coef- ficient magnitudes of different eigenstates are plotted against each other-Fig. 4(b) shows that low/high-energy eigenstates have anomalously large weights in a few basis states, which have small weights in midspectrum eigenstates [39].

In Fig. 4(c) we show how orthogonality affects entanglement. Starting from a random state, we successively Gram-Schmidt orthogonalize against pairs of eigenstates at the outermost edges of the spectrum, i.e., first with the lowest and highest eigenstates, then with the second-lowest and second-highest eigenstates, and so on. The EE (both block and comb) of the resulting states, Fig. 4(c), start with zero departure and, after all but the midspectrum eigenstates have been orthogonalized away, end at roughly the values observed for the EE's of midspectrum eigenstates. The curve has fluctuations depending on the initial random state used, but the fluctuations decrease with increasing system size and the overall picture is valid for a variety of random states tried [39]. When averaged over various realizations of the starting random state, one obtains a relatively smooth curve, as shown in the inset.

For simplicity, we have above described the blockade as being due to only the eigenstates at the spectral edges. In truth, the midspectrum eigenstates are affected by orthogonality to all nonmidspectrum eigenstates, not just those at the spectral edges. Intuitively, one might expect the spectral edges to have the strongest effect, as these eigenstates are the least generic or random-like. Comparing with the straight line in the inset to Fig. 4(c), we see that the slope of the curve is larger for small $k$, i.e., orthogonalizing against the spectral edges has a stronger effect than orthogonalizing against eigenstates which are intermediate between the spectral edges and the midspectrum region. This demonstrates that eigenstates closer to the spectral edges indeed have a stronger role in causing the midspectrum departure [39]. For simplicity, we sometimes loosely describe the blockade to be due to spectral edge states-it should be understood that intermediate states also contribute to the phenomenon, albeit to a weaker degree.

Interestingly, the distribution of coefficients by itself does not predict the correct midspectrum EE. Using a random state with coefficients drawn from the observed midspectrum distribution, Fig. 4(a), we find the resulting EE to have a departure one order of magnitude smaller than that observed in the comb cases. Thus, a random state with an effective Hilbert space of reduced dimension $D_{\text {eff }}<D$ (as in Ref. [55]) is not sufficient to model the departure. The orthogonality causes very particular combinations of configurations to be missing from the midspectrum eigenstates; this "correlated blockade" is necessary for the observed departure [39].

\section{RAINBOW SHAPE IMPLIES DEPARTURE}

The orthogonality mechanism has the following implication. If the EE versus eigenenergy plot is rainbow- or arch-shaped, then the correlations in spectral-edge eigenstates which cause those to have low entanglement will affect the midspectrum eigenstates by orthogonality, causing the midspectrum eigenstates to depart from Gaussian randomness. Thus, a rainbow shape is necessarily accompanied by a departure in the midspectrum states. 

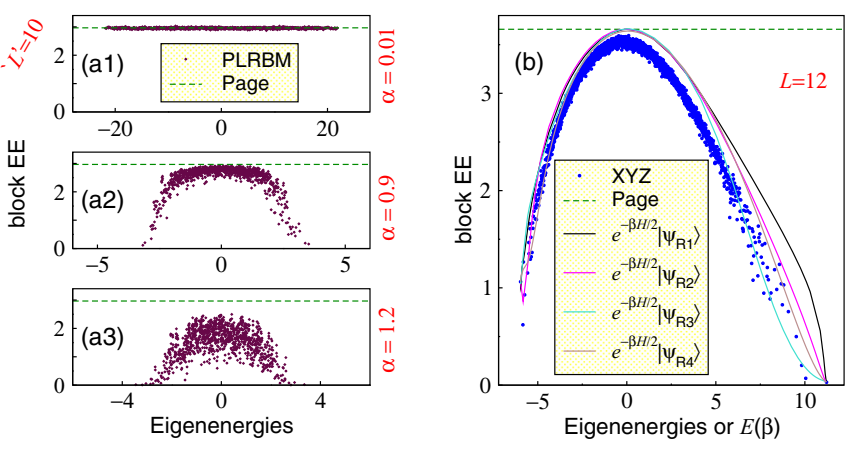

FIG. 5. (a) Power-law random banded matrix of size $2^{10}$, treated as a 10-spin Hamiltonian. EE versus eigenvalues, for exponent $\alpha$ in the ergodic/delocalized (a1), weakly ergodic (a2) and localized (a3) regimes [39]. Case (a2) is analogous to the many-body situation. (b) Lines are block EE calculated from states $e^{-\frac{1}{2} \beta H}\left|\psi_{R}\right\rangle$, for different realizations of random $\left|\psi_{R}\right\rangle$. On average, these lie above actual eigenstate EE's at all temperatures/energies.

We can thus trace back the departure for comb EE to the fact that the EE plot is rainbow-shaped for comb bipartitioning. Unlike block partitioning, there is now no parametric argument $\left(\sim L^{0}\right.$ vs $\left.\sim L^{1}\right)$ for the rainbow shape, as the spectral edge EE now scales as $\sim L$ (the scaling of the boundary between partitions), the same as the midspectrum EE. A general argument for the rainbow shape is that, because the low-/high-energy states are more constrained (less like random states) compared to midspectrum eigenstates, the EE at spectral edges has to be farther from the Page value compared to midspectrum EE. However, this is not obviously related to the spatial structure of correlations. In fact, in a model without spatial locality, where all eigenstates have volumelaw scaling, the EE is found to also have a rainbow structure [40]. According to the picture presented above, orthogonality should then force a departure of the midspectrum EE; indeed this is observed [40].

Our picture is applicable also beyond the context of many-body physics. In power-law random banded matrices (PLRBMs) [49,56-66] and ultrametric matrices $[61-64,67,68]$, there is a regime of parameters where the midspectrum eigenstates are "weakly ergodic" in the sense that, even though the scaling with matrix size matches the ergodic case, there is deviation from GOE/GUE ensembles at any finite size $[44,64,66]$. To connect to the present topic: by interpreting the indices of such matrices as spatial configurations (as discussed in, e.g., Refs. [39,69,70]), one can evaluate entanglements. For PLRBMs in the weakly ergodic regime, we have found a rainbow-shaped dependence of EE versus eigenenergy, with a midspectrum departure (Fig. 5(a) and [39]), just as in the many-body case. The spectral edges are likely power-law-localized [39], so that the blockade effect is more direct than in the many-body situation.

\section{CONTEXT AND CONSEQUENCES}

The EE of nonextremal eigenstates is now the focus of considerable attention, both for chaotic systems $[22,25,26,40,71-$ 87] as in this work, and also for integrable systems $[25,40,41,70,88-102]$. The eigenstate EE plays a role in connecting quantum properties to the thermodynamic entropy $[9,26,72-74,80,92,103]$. Current theory suggests that the midspectrum states are effectively random. We have shown that a subleading deviation is present for any finite size, and have developed concepts (orthogonality blockade, residual spatial correlations) pertinent to understanding this deviation. We expect our results to be equally valid for systems with symmetries (for which the average random-state $\mathrm{EE}$ is not given by the Page formula), and that the presented concepts will have further applications, e.g., effects of orthogonality to the extremal eigenstates has been exploited in recent literature [66,104-106]. An open question is whether the deviation saturates or vanishes in the large-size limit.

The idea that the state $e^{-\frac{1}{2} \beta H}\left|\psi_{R}\right\rangle$ (with $\left|\psi_{R}\right\rangle$ a random state) is a good model for finite-temperature eigenstates $[17,20,22]$ has been fruitful for numerical computations of thermodynamic properties [107-118]. Our observation, that midspectrum (highest-EE or infinite-temperature) eigenstates show departures from random-state properties, implies that the state $e^{-\frac{1}{2} \beta H}\left|\psi_{R}\right\rangle$ is an imperfect model for lowerentanglement (finite-temperature) eigenstates as well. In fact, one can attempt to reproduce the entanglement rainbow by plotting the EE of the state $e^{-\frac{1}{2} \beta H}\left|\psi_{R}\right\rangle$ against the corresponding energy. We find that this curve falls systematically above the EE scatter-plot of actual eigenstates (Fig. 5(b) and Ref. [39]).

The departure is a signature of deviation from GOE/GUE behavior at all finite sizes. Signatures of this deviation also appear in eigenstate coefficient distributions (Fig. 4(a); also Refs. [42-45]). midspectrum eigenstates have the same scaling behavior as GOE/GUE, but approach the thermodynamic limit differently, as also seen in multifractality analysis [44]. This behavior could justifiably be called "weakly ergodic," although the phrase does not yet have a widely accepted definition $[44,64,66,104,119,120]$. In Ref. [120], weak ergodicity is associated with breaking of "basis rotation invariance." Our finding, that midspectrum eigenstates have different block EE and comb $\mathrm{EE}$, is another type of noninvariance under basis rotation.

Note added in proof. Recently, we became aware of Ref. [121], which quantitatively accounts for part of the departure for block partitions, in the case where the Hamiltonian is local.

\section{ACKNOWLEDGMENTS}

We thank A. Bäcker and G. De Tomasi for useful discussions. The work of I.M.K. is supported by the Russian Science Foundation under the Grant No. 21-12-00409.
[1] J. M. Deutsch, Quantum statistical mechanics in a closed system, Phys. Rev. A 43, 2046 (1991).
[2] M. Srednicki, Chaos and quantum thermalization, Phys. Rev. E 50, 888 (1994). 
[3] M. Rigol, V. Dunjko, and M. Olshanii, Thermalization and its mechanism for generic isolated quantum systems. Nature (London) 452, 854 (2008).

[4] P. Reimann, Eigenstate thermalization: Deutsch's approach and beyond, New J. Phys. 17, 055025 (2015).

[5] L. D'Alessio, Y. Kafri, A. Polkovnikov, and M. Rigol, From quantum chaos and eigenstate thermalization to statistical mechanics and thermodynamics, Adv. Phys. 65, 239 (2016).

[6] J. M. Deutsch, Eigenstate thermalization hypothesis, Rep. Prog. Phys. 81, 082001 (2018).

[7] T. Mori, T. N. Ikeda, E. Kaminishi, and M. Ueda, Thermalization and prethermalization in isolated quantum systems: A theoretical overview, J. Phys. B: Atom. Mol. Opt. Phys. 51, 112001 (2018).

[8] H. Tasaki, From Quantum Dynamics to the Canonical Distribution: General Picture and a Rigorous Example, Phys. Rev. Lett. 80, 1373 (1998).

[9] J. Gemmer, A. Otte, and G. Mahler, Quantum Approach to a Derivation of the Second Law of Thermodynamics, Phys. Rev. Lett. 86, 1927 (2001).

[10] S. Goldstein, J. L. Lebowitz, R. Tumulka, and N. Zanghì, Canonical Typicality, Phys. Rev. Lett. 96, 050403 (2006).

[11] S. Popescu, A. J Short, and A. Winter, Entanglement and the foundations of statistical mechanics, Nat. Phys. 2, 754 (2006).

[12] A. Sugita, On the basis of quantum statistical mechanics, Nonlin. Phenom. Complex Syst. 10, 192 (2007).

[13] P. Reimann, Typicality for Generalized Microcanonical Ensembles, Phys. Rev. Lett. 99, 160404 (2007).

[14] J. Gemmer, M. Michel, and G. Mahler, Quantum Thermodynamics: Emergence of Thermodynamic Behavior Within Composite Quantum Systems, Lecture Notes in Physics, Vol. 784 (Springer, Berlin, 2009).

[15] S. Goldstein, J. L. Lebowitz, R. Tumulka, and N. Zanghi, Long-time behavior of macroscopic quantum systems, Eur. Phys. J. H 35, 173 (2010).

[16] J. von Neumann, Proof of the ergodic theorem and the htheorem in quantum mechanics, Eur. Phys. J. H 35, 201 (2010).

[17] S. Sugiura and A. Shimizu, Canonical Thermal Pure Quantum State, Phys. Rev. Lett. 111, 010401 (2013).

[18] H. Tasaki, Typicality of thermal equilibrium and thermalization in isolated macroscopic quantum systems, J. Stat. Phys. 163, 937 (2016).

[19] S. R. White, Minimally Entangled Typical Quantum States at Finite Temperature, Phys. Rev. Lett. 102, 190601 (2009).

[20] S. Sugiura and A. Shimizu, Thermal Pure Quantum States at Finite Temperature, Phys. Rev. Lett. 108, 240401 (2012).

[21] T. A. Elsayed and B. V. Fine, Regression Relation for Pure Quantum States and Its Implications for Efficient Computing, Phys. Rev. Lett. 110, 070404 (2013).

[22] Y. O. Nakagawa, M. Watanabe, H. Fujita, and S. Sugiura, Universality in volume-law entanglement of scrambled pure quantum states, Nat. Commun. 9, 1635 (2018).

[23] J. Eisert, M. Cramer, and M. B. Plenio, Colloquium: Area laws for the entanglement entropy, Rev. Mod. Phys. 82, 277 (2010).

[24] L. Amico, R. Fazio, A. Osterloh, and V. Vedral, Entanglement in many-body systems, Rev. Mod. Phys. 80, 517 (2008).
[25] W. Beugeling, A. Andreanov, and M. Haque, Global characteristics of all eigenstates of local many-body Hamiltonians: Participation ratio and entanglement entropy, J. Stat. Mech.: Theory Exp. (2015) P02002.

[26] J. R. Garrison and T. Grover, Does a Single Eigenstate Encode the Full Hamiltonian? Phys. Rev. X 8, 021026 (2018).

[27] C. J. Turner, A. A. Michailidis, D. A. Abanin, M. Serbyn, and Z. Papić, Quantum scarred eigenstates in a Rydberg atom chain: Entanglement, breakdown of thermalization, and stability to perturbations, Phys. Rev. B 98, 155134 (2018).

[28] S. Moudgalya, N. Regnault, and B. A. Bernevig, Entanglement of exact excited states of Affleck-Kennedy-Lieb-Tasaki models: Exact results, many-body scars, and violation of the strong eigenstate thermalization hypothesis, Phys. Rev. B 98, 235156 (2018).

[29] S. Ok, K. Choo, C. Mudry, C. Castelnovo, C. Chamon, and T. Neupert, Topological many-body scar states in dimensions one, two, and three, Phys. Rev. Research 1, 033144 (2019).

[30] B. Mukherjee, S. Nandy, A. Sen, D. Sen, and K. Sengupta, Collapse and revival of quantum many-body scars via Floquet engineering, Phys. Rev. B 101, 245107 (2020).

[31] B. Mukherjee, A. Sen, D. Sen, and K. Sengupta, Dynamics of the vacuum state in a periodically driven Rydberg chain, Phys. Rev. B 102, 075123 (2020).

[32] T. Iadecola and M. Schecter, Quantum many-body scar states with emergent kinetic constraints and finite-entanglement revivals, Phys. Rev. B 101, 024306 (2020).

[33] D. K. Mark, C.-J. Lin, and O. I. Motrunich, Unified structure for exact towers of scar states in the Affleck-Kennedy-LiebTasaki and other models, Phys. Rev. B 101, 195131 (2020).

[34] N. Shibata, N. Yoshioka, and H. Katsura, Onsager's Scars in Disordered Spin Chains, Phys. Rev. Lett. 124, 180604 (2020).

[35] S. Chattopadhyay, H. Pichler, M. D. Lukin, and W. W. Ho, Quantum many-body scars from virtual entangled pairs, Phys. Rev. B 101, 174308 (2020).

[36] D. K. Mark and O. I. Motrunich, $\eta$-pairing states as true scars in an extended Hubbard model, Phys. Rev. B 102, 075132 (2020).

[37] P. A. McClarty, M. Haque, A. Sen, and J. Richter, Disorderfree localization and many-body quantum scars from magnetic frustration, Phys. Rev. B 102, 224303 (2020).

[38] D. N. Page, Average Entropy of a Subsystem, Phys. Rev. Lett. 71, 1291 (1993).

[39] See Supplemental Material at http://link.aps.org/ supplemental/10.1103/PhysRevE.105.014109 for further details and additional supporting data.

[40] C. Liu, X. Chen, and L. Balents, Quantum entanglement of the Sachdev-Ye-Kitaev models, Phys. Rev. B 97, 245126 (2018).

[41] T. LeBlond, K. Mallayya, L. Vidmar, and M. Rigol, Entanglement and matrix elements of observables in interacting integrable systems, Phys. Rev. E 100, 062134 (2019).

[42] W. Beugeling, A. Bäcker, R. Moessner, and M. Haque, Statistical properties of eigenstate amplitudes in complex quantum systems, Phys. Rev. E 98, 022204 (2018).

[43] D. J. Luitz and Y. Bar Lev, Anomalous Thermalization in Ergodic Systems, Phys. Rev. Lett. 117, 170404 (2016).

[44] A. Bäcker, M. Haque, and I. M. Khaymovich, Multifractal dimensions for random matrices, chaotic quantum maps, and many-body systems, Phys. Rev. E 100, 032117 (2019). 
[45] D. J. Luitz, I. M. Khaymovich, and Y. B. Lev, Multifractality and its role in anomalous transport in the disordered $X X Z$ spin-chain, SciPost Phys. Core 2, 6 (2020).

[46] B. M. McCoy, Advanced Statistical Mechanics, International series of monographs on physics (Oxford University Press, Oxford, UK, 2009).

[47] P. Vivo, M. P. Pato, and G. Oshanin, Random pure states: Quantifying bipartite entanglement beyond the linear statistics, Phys. Rev. E 93, 052106 (2016).

[48] G. De Tomasi, S. Bera, J. H. Bardarson, and F. Pollmann, Quantum Mutual Information as a Probe for Many-Body Localization, Phys. Rev. Lett. 118, 016804 (2017).

[49] G. De Tomasi, Algebraic many-body localization and its implications on information propagation, Phys. Rev. B 99, 054204 (2019).

[50] J. P. Keating, F. Mezzadri, and M. Novaes, Comb entanglement in quantum spin chains, Phys. Rev. A 74, 012311 (2006).

[51] Y. Chen, P. Zanardi, Z. D. Wang, and F. C. Zhang, Sublattice entanglement and quantum phase transitions in antiferromagnetic spin chains, New J. Phys. 8, 97 (2006).

[52] F. Iglói and I. Peschel, On reduced density matrices for disjoint subsystems, Europhys. Lett. 89, 40001 (2010).

[53] R. Rossignoli, N. Canosa, and J. M. Matera, Even-odd entanglement in boson and spin systems, Phys. Rev. A 83, 042328 (2011).

[54] T. He, J. M. Magán, and S. Vandoren, Entanglement entropy of periodic sublattices, Phys. Rev. B 95, 035130 (2017).

[55] G. De Tomasi and I. M. Khaymovich, Multifractality Meets Entanglement: Relation for Nonergodic Extended States, Phys. Rev. Lett. 124, 200602 (2020).

[56] A. D. Mirlin, Y. V. Fyodorov, F.-M. Dittes, J. Quezada, and T. H. Seligman, Transition from localized to extended eigenstates in the ensemble of power-law random banded matrices, Phys. Rev. E 54, 3221 (1996).

[57] V. E. Kravtsov and K. A. Muttalib, New Class of Random Matrix Ensembles with Multifractal Eigenvectors, Phys. Rev. Lett. 79, 1913 (1997).

[58] I. Varga and D. Braun, Critical statistics in a power-law random-banded matrix ensemble, Phys. Rev. B 61, R11859 (2000).

[59] A. D. Mirlin and F. Evers, Multifractality and critical fluctuations at the anderson transition, Phys. Rev. B 62, 7920 (2000).

[60] F. Evers and A. D. Mirlin, Anderson transitions, Rev. Mod. Phys. 80, 1355 (2008).

[61] E. Bogomolny and O. Giraud, Eigenfunction Entropy and Spectral Compressibility for Critical Random Matrix Ensembles, Phys. Rev. Lett. 106, 044101 (2011).

[62] I. Rushkin, A. Ossipov, and Y. V. Fyodorov, Universal and nonuniversal features of the multifractality exponents of critical wavefunctions, J. Stat. Mech.: Theory Exp. (2011) L03001.

[63] J. A. Mendez-Bermudez, A. Alcazar-Lopez, and I. Varga, Multifractal dimensions for critical random matrix ensembles, Europhys. Lett. 98, 37006 (2012).

[64] E. Bogomolny and M. Sieber, Power-law random banded matrices and ultrametric matrices: Eigenvector distribution in the intermediate regime, Phys. Rev. E 98, 042116 (2018).

[65] D. A. Vega-Oliveros, J. A. Méndez-Bermúdez, and F. A. Rodrigues, Multifractality in random networks with powerlaw decaying bond strengths, Phys. Rev. E 99, 042303 (2019).
[66] P. A. Nosov, I. M. Khaymovich, and V. E. Kravtsov, Correlation-induced localization, Phys. Rev. B 99, 104203 (2019).

[67] Y. V. Fyodorov, A. Ossipov, and A. Rodriguez, The anderson localization transition and eigenfunction multifractality in an ensemble of ultrametric random matrices, J. Stat. Mech.: Theory Exp. (2009) L12001.

[68] P. von Soosten and S. Warzel, The localization transition in the ultrametric ensemble, arXiv preprint arXiv:1705.04165 (2017).

[69] I. M. Khaymovich, M. Haque, and P. A. McClarty, Eigenstate Thermalization, Random Matrix Theory, and Behemoths, Phys. Rev. Lett. 122, 070601 (2019).

[70] P. Łydżba, M. Rigol, and L. Vidmar, Eigenstate Entanglement Entropy in Random Quadratic Hamiltonians, Phys. Rev. Lett. 125, 180604 (2020).

[71] C. Mejía-Monasterio, G. Benenti, G. G. Carlo, and G. Casati, Entanglement across a transition to quantum chaos, Phys. Rev. A 71, 062324 (2005).

[72] J. M. Deutsch, Thermodynamic entropy of a many-body energy eigenstate, New J. Phys. 12, 075021 (2010).

[73] L. F. Santos, A. Polkovnikov, and M. Rigol, Weak and strong typicality in quantum systems, Phys. Rev. E 86, 010102(R) (2012).

[74] J. M. Deutsch, H. Li, and A. Sharma, Microscopic origin of thermodynamic entropy in isolated systems, Phys. Rev. E 87, 042135 (2013).

[75] L. Zhang, H. Kim, and D. A. Huse, Thermalization of entanglement, Phys. Rev. E 91, 062128 (2015).

[76] L. Vidmar and M. Rigol, Entanglement Entropy of Eigenstates of Quantum Chaotic Hamiltonians, Phys. Rev. Lett. 119, 220603 (2017).

[77] A. Dymarsky, N. Lashkari, and H. Liu, Subsystem eigenstate thermalization hypothesis, Phys. Rev. E 97, 012140 (2018).

[78] H. Fujita, Y. O Nakagawa, S. Sugiura, and M. Watanabe, Page curves for general interacting systems, J. High Energy Phys. 12 (2018) 112.

[79] M. Fremling, C. Repellin, J.-M. Stéphan, N. Moran, J. K. Slingerland, and M. Haque, Dynamics and level statistics of interacting fermions in the lowest Landau level, New J. Phys. 20, 103036 (2018).

[80] C. Murthy and M. Srednicki, Structure of chaotic eigenstates and their entanglement entropy, Phys. Rev. E 100, 022131 (2019).

[81] H. Wilming, M. Goihl, I. Roth, and J. Eisert, EntanglementErgodic Quantum Systems Equilibrate Exponentially Well, Phys. Rev. Lett. 123, 200604 (2019).

[82] Y. Huang, Universal eigenstate entanglement of chaotic local Hamiltonians, Nucl. Phys. B 938, 594-604 (2019).

[83] Y. Huang and Y. Gu, Eigenstate entanglement in the SachdevYe-Kitaev model, Phys. Rev. D 100, 041901(R) (2019).

[84] T.-C. Lu and T. Grover, Renyi entropy of chaotic eigenstates, Phys. Rev. E 99, 032111 (2019).

[85] Q. Miao and T. Barthel, Eigenstate Entanglement: Crossover from the Ground State to Volume Laws, Phys. Rev. Lett. 127, 040603 (2021).

[86] S. C. Morampudi, A. Chandran, and C. R. Laumann, Universal Entanglement of Typical States in Constrained Systems, Phys. Rev. Lett. 124, 050602 (2020). 
[87] K. Kaneko, E. Iyoda, and T. Sagawa, Characterizing complexity of many-body quantum dynamics by higherorder eigenstate thermalization, Phys. Rev. A 101, 042126 (2020).

[88] V. Alba, M. Fagotti, and P. Calabrese, Entanglement entropy of excited states, J. Stat. Mech. (2009) P10020.

[89] F. C. Alcaraz, M. I. Berganza, and G. Sierra, Entanglement of Low-Energy Excitations in Conformal Field Theory, Phys. Rev. Lett. 106, 201601 (2011).

[90] J. Mölter, T. Barthel, U. Schollwöck, and V. Alba, Bound states and entanglement in the excited states of quantum spin chains, J. Stat. Mech.: Theory Exp. (2014) P10029.

[91] F. Ares, J. G. Esteve, F. Falceto, and E. Sánchez-Burillo, Excited state entanglement in homogeneous fermionic chains, J. Phys. A: Math. Theor. 47, 245301 (2014).

[92] M. Storms and R. R. P. Singh, Entanglement in ground and excited states of gapped free-fermion systems and their relationship with fermi surface and thermodynamic equilibrium properties, Phys. Rev. E 89, 012125 (2014).

[93] H.-H. Lai and K. Yang, Entanglement entropy scaling laws and eigenstate typicality in free fermion systems, Phys. Rev. B 91, 081110(R) (2015).

[94] V. Alba, Eigenstate thermalization hypothesis and integrability in quantum spin chains, Phys. Rev. B 91, 155123 (2015).

[95] S. Nandy, A. Sen, A. Das, and A. Dhar, Eigenstate gibbs ensemble in integrable quantum systems, Phys. Rev. B 94, 245131 (2016).

[96] L. Vidmar, L. Hackl, E. Bianchi, and M. Rigol, Entanglement Entropy of Eigenstates of Quadratic Fermionic Hamiltonians, Phys. Rev. Lett. 119, 020601 (2017).

[97] L. Vidmar, L. Hackl, E. Bianchi, and M. Rigol, Volume Law and Quantum Criticality in the Entanglement Entropy of Excited Eigenstates of the Quantum Ising Model, Phys. Rev. Lett. 121, 220602 (2018).

[98] Y. Zhang, L. Vidmar, and M. Rigol, Information measures for a local quantum phase transition: Lattice fermions in a one-dimensional harmonic trap, Phys. Rev. A 97, 023605 (2018).

[99] O. A. Castro-Alvaredo, C. De Fazio, B. Doyon, and I. M. Szécsényi, Entanglement Content of Quasiparticle Excitations, Phys. Rev. Lett. 121, 170602 (2018).

[100] L. Hackl, L. Vidmar, M. Rigol, and E. Bianchi, Average eigenstate entanglement entropy of the $X Y$ chain in a transverse field and its universality for translationally invariant quadratic fermionic models, Phys. Rev. B 99, 075123 (2019).

[101] A. Jafarizadeh and M. A. Rajabpour, Bipartite entanglement entropy of the excited states of free fermions and harmonic oscillators, Phys. Rev. B 100, 165135 (2019).

[102] T. Barthel and Q. Miao, Scaling functions for eigenstate entanglement crossovers in harmonic lattices, Phys. Rev. A 104, 022414 (2021).

[103] E. Iyoda, K. Kaneko, and T. Sagawa, Fluctuation Theorem for Many-Body Pure Quantum States, Phys. Rev. Lett. 119, 100601 (2017).

[104] P. A. Nosov and I. M. Khaymovich, Robustness of delocalization to the inclusion of soft constraints in long-range random models, Phys. Rev. B 99, 224208 (2019).
[105] A. G. Kutlin and I. M. Khaymovich, Renormalization to localization without a small parameter, SciPost Phys. 8, 49 (2020).

[106] X. Deng, A. L. Burin, and I. M. Khaymovich, Anisotropymediated reentrant localization (2020), arXiv:2002.00013.

[107] R. Steinigeweg, J. Gemmer, and W. Brenig, Spin-Current Autocorrelations from Single Pure-State Propagation, Phys. Rev. Lett. 112, 120601 (2014).

[108] T. Monnai and A. Sugita, Typical pure states and nonequilibrium processes in quantum many-body systems, J. Phys. Soc. Jpn. 83, 094001 (2014).

[109] R. Steinigeweg, J. Gemmer, and W. Brenig, Spin and energy currents in integrable and nonintegrable spin- $\frac{1}{2}$ chains: A typicality approach to real-time autocorrelations, Phys. Rev. B 91, 104404 (2015).

[110] F. Jin, R. Steinigeweg, F. Heidrich-Meisner, K. Michielsen, and H. De Raedt, Finite-temperature charge transport in the one-dimensional Hubbard model, Phys. Rev. B 92, 205103 (2015).

[111] R. Steinigeweg, J. Herbrych, F. Pollmann, and W. Brenig, Typicality approach to the optical conductivity in thermal and many-body localized phases, Phys. Rev. B 94, 180401(R) (2016).

[112] H. Endo, C. Hotta, and A. Shimizu, From Linear to Nonlinear Responses of Thermal Pure Quantum States, Phys. Rev. Lett. 121, 220601 (2018).

[113] K. Inoue, Y. Maeda, H. Nakano, and Y. Fukumoto, Canonicalensemble calculations of the magnetic susceptibility for a spin-1/2 spherical kagome cluster with DzyaloshinskiiMoriya interactions by using microcanonical thermal pure quantum states, IEEE Trans. Magn. 55, 1 (2019).

[114] J. Richter and R. Steinigeweg, Combining dynamical quantum typicality and numerical linked cluster expansions, Phys. Rev. B 99, 094419 (2019).

[115] I. Rousochatzakis, S. Kourtis, J. Knolle, R. Moessner, and N. B. Perkins, Quantum spin liquid at finite temperature: Proximate dynamics and persistent typicality, Phys. Rev. B 100, 045117 (2019).

[116] J. Schnack, J. Richter, and R. Steinigeweg, Accuracy of the finite-temperature Lanczos method compared to simple typicality-based estimates, Phys. Rev. Research 2, 013186 (2020).

[117] H. Nishida, R. Fujiuchi, K. Sugimoto, and Y. Ohta, Typicalitybased variational cluster approach to thermodynamic properties of the Hubbard model, J. Phys. Soc. Jpn. 89, 023702 (2020).

[118] J. Schnack, J. Richter, T. Heitmann, J. Richter, and R. Steinigeweg, Finite-size scaling of typicality-based estimates, Z. Naturforsch. A 75, 465 (2020).

[119] G. De Tomasi, S. Bera, A. Scardicchio, and I. M. Khaymovich, Subdiffusion in the Anderson model on the random regular graph, Phys. Rev. B 101, 100201(R) (2020).

[120] I. M. Khaymovich, V. E. Kravtsov, B. L. Altshuler, and L. B. Ioffe, Fragile extended phases in the log-normal RosenzweigPorter model, Phys. Rev. Research 2, 043346 (2020).

[121] Y. Huang, Universal entanglement of mid-spectrum eigenstates of chaotic local Hamiltonians, Nucl. Phys. B 966, 115373 (2021). 